Journal of Measurements, Electronics, Communications, and Systems (Vol.04, Pg.8-15, June 2018)

\title{
Prototype of Dual DDS FMCW Transmitter for L-Band Synthetic Aperture Radar
}

\author{
Edwar*1, A. Munir $^{2}$ \\ ${ }^{1}$ School of Electrical Engineering, Telkom University \\ ${ }^{2}$ School of Electrical Engineering and Informatics, Bandung Institute of Technology \\ *edwarm@telkomuniversity.ac.id
}

Manuscript received January 19, 2018; revised April 10, 2018; accepted Mei 2, 2018

\begin{abstract}
One of the popular remote sensing technique is remote sensing using radar technology such as Synthetic Aperture Radar (SAR). In this paper, a compact SAR prototype transmitter was designed so it could be installed in small platform. This SAR transmitter was designed to generate a frequency modulated continuous wave (FMCW) using direct digital synthesizer (DDS) integrated with RF front end modules such as analog filter and power amplifier. The bandwidth of the radar spectrum is $10 \mathrm{MHz}$, and the carrier frequency used is $1.27 \mathrm{GHz}$. The L-Band has been chosen as the carrier signal in order to detect the target (trees). The payload was designed for aerial vehicle, hence the choice of components should be as small as possible. The FMCW-SAR transmitter was implemented by using DDS module AD9850 and integrated with another RF component. The FMCW-SAR transmitter produces the transmit power about $-17.67 \mathrm{dBm}$.
\end{abstract}

Keywords: Remote Sensing; SAR; FMCW; DDS

DOI: https://doi.org/10.25124/jmecs.v4i1.1694

\section{Introduction}

Active remote sensing, such as radar, is a technique to obtain object's properties by illuminating the target with electromagnetic wave or laser, receiving its reflection, then proceeding the reflected signal into a set of information [1]. Synthetic Aperture Radar (SAR) is a popular radar technology that has been learned and developed to sense the remote target such as volcanoes, flood, air surveillance and many more applications [2-4]. There are few advantages of using radar to observe the environment, for example, radar can be operated in day or night because it is not dependent on sun light. Moreover, for airborne and spaceborne radar, it can also can detect targets even though clouds cover them [5]. SAR technology is able to increase the cross-resolution of common radar target area [6] and it is mostly installed in an moving platform such as UAV (Unmanned Aerial Vehicle). That platform generates limited electrical power and has restriction on payload size.
FMCW radar is the right answer for SAR application that has limitation on its power and dimension. FMCW transmits continuous wave which naturally consumes smaller power because its spectral density is widely spread in its spectrum. In addition, the FMCW also emits continuous wave that has been modulated, where the frequency modulation is popular technique to use in this case. This method is applied to solve the natural problem of continuous wave radar that is not able to detect the target range by giving a timing mark to the transmitted signal. From the circuit view, FMCW radar can be built with simple design that consists a less number of components which makes this technology fit with SAR in moving platform such as UAV or satellite.

In this research, the SAR transmitter has been designed to be as compact as possible to emit FMCW radar signal in L-Band using dual DDS. The transmitter design was restricted by few matters, such as small component selection, low power 
Edwar et al / Journal of Measurement, Electronics and Communication Systems

consumption, and FMCW signal above the minimum specification of this remote sensing mission.

\section{Theory Overview}

\section{2..1 Radar Technology}

Radar (Radio Detection and Ranging) is an active remote sensing device that use electromagnetic wave to detect the target. Firstly radar had been invented as a part of the war equipment [7]. Recently, many researchers use radar ability to detect target in many applications such as bridge health monitoring, through wall target detection, forest and land monitoring [8], etc. Radar works by emitting electromagnetic wave to the target, receiving its reflection, then gathering the object's informations through signal processing process. The Fig. 1 shows the simple radar system block, which contains signal generator, the antennas, and the digital processor, and how it works.

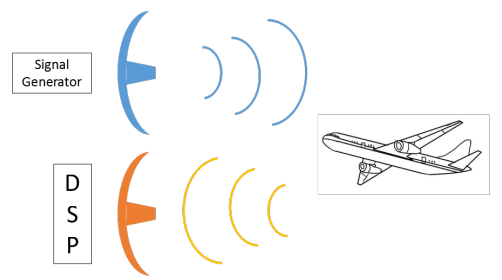

Fig. 1. How Radar Works

\section{2..2 FMCW-SAR Characteristic and Performance}

FMCW SAR (Frequency Modulated Continuous Wave Synthetic Aperture Radar) is a type of continuous wave radar which is using modulated wave to detect the object.[9] This radar fix the ordinary $\mathrm{CW}$ radar flaw, the inability to get the target's range information. The 'Synthetic' term describes that the radar form an artificial aperture by using the small antenna that is installed in moving platform such as aerial vehicle or spacecraft. The Fig. 2 describes the shape of the triangular FMCW waveform and the Fig. 3 shows how a SAR works.

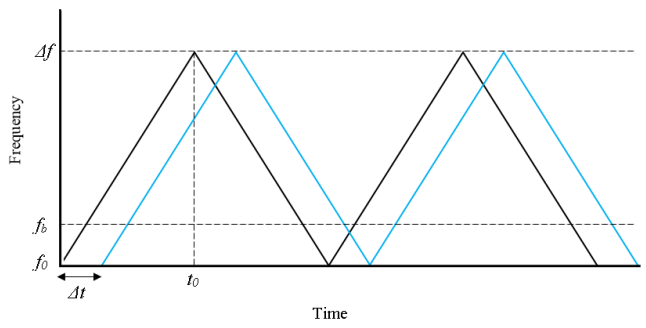

Fig. 2. FMCW radar waveform

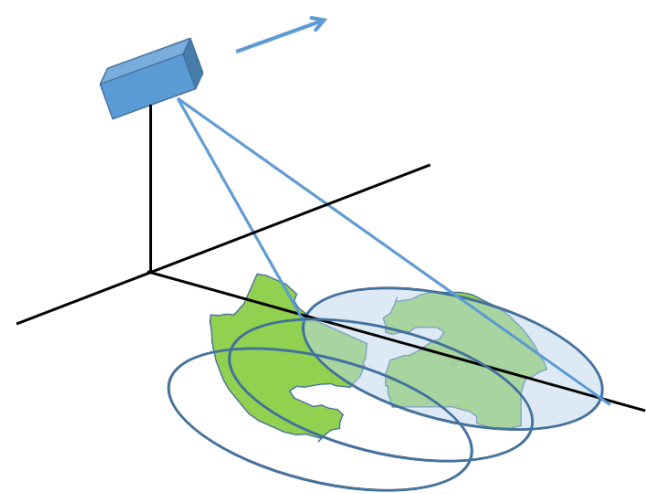

Fig. 3. Description of SAR work

At the Fig. 2, the black line represent the transmitted FMCW signal and the blue line represent the reflection signal that is received. There is delay between both transmitted and received signal as long as $\Delta t$. Variable $t_{0}$ is the time needed by the FMCW chirp generator to produce the FMCW signal from the start frequency $\left(f_{0}\right)$ up to the stop frequency with the frequency deviation $(\Delta f)$. To determine the target range and velocity, the difference between transmitted and received signals is used to yield the frequency beat $\left(f_{b}\right)$. For the stationary target, the target's distance $R$ is determined by using Eq.(1) [10].

$$
R=\frac{c f_{b}}{2 \dot{f}}
$$

$c$ is the speed of light $\left(3 \times 10^{8} \mathrm{~m} / \mathrm{s}\right)$. The symbol $\dot{f}$ is the frequency rate, which is obtained from Eq.(2) [20].

$$
\dot{f}=\frac{\Delta f}{t_{0}}
$$

In the Fig. 3, the antenna's beam is set to be perpendicular with the aircraft path. From this condition, we could identify that the range resolution is also perpendicular against the observation track and the cross-range resolution will be parallel with the track. The range resolution for SAR is not different with common radar, and SAR technique gives no effect to this resolution. As mentioned before, SAR only focus is to make the cross-range resolution of a radar getting better than RAR.

In RAR (Real Aperture Radar), cross-range resolution can be achieved by using Equation (3). However, the cross-range resolution in $\operatorname{SAR}\left(\delta_{c r}\right)$ can be obtained by modified the Eq.(3) where the distance variable $D$ is replaced with the long synthetic aperture track $L_{S A}$ like showed in Eq.(4)[11].

$$
\delta_{c r}=\frac{R \lambda}{D}
$$


Edwar et al / Journal of Measurement, Electronics and Communication Systems

$$
\delta_{c r}=\frac{R \lambda}{2 L_{S A}}=\frac{\lambda}{2 \Delta \theta}
$$

With $\Delta \theta$ is the synthetic aperture angle which is formed from the position of the observer with the target.

Next, for the SAR range resolution value, will be influence by how wide the bandwidth system $(B)$ used. The range resolution can be calculated by using Eq.(5)[11]

$$
\Delta R=\frac{c}{2 B}
$$

In order to measure the performance of the SAR system, there are some parameters which are need to be defined. The performance can be calculated by using Eq.(6)[11].

$$
S N R=\frac{P_{t} G^{2} \lambda^{2} \sigma}{(2 \pi)^{3} k T_{0} F L R^{4}}
$$

Due to SAR using small antenna and it is moved to sweep the observation area as far as $L_{S A}$, the time factor $t_{a}$, time needed to complete the sweeping proces, will affect the Equation (6). The result, the equation will be modified by multiplying the Equation (6) with $t_{a}$ become Eq.(7) [11].

$$
S N R=\frac{P_{t} G^{2} \lambda^{2} \sigma}{(4 \pi)^{3} k T_{0} F L} t_{a}
$$

With $t_{a}$ meets the Eq.(8) [11]

$$
t_{a}=\frac{\lambda R}{2 V \delta_{c r}}
$$

Radar Cross Section (RCS) $\sigma$ used in Eq.(7) is the RCS per unit area $\left(\sigma^{0}\right)$, which is related with the RCS of target. Variable $\sigma^{0}$ is derived from Eq.(9) [11].

$$
\sigma=\frac{\sigma^{0} \delta_{c r} \delta_{r}}{\cos \psi}
$$

by applying substitution towards variable $t_{a}$ and $\sigma$ in Eq.(6) with the Eq.(8) and Eq.(9), then will be obtained the final equation to calculate the SAR permormance by Eq.(10)[11].

$$
S N R=\frac{P_{t} G^{2} \lambda^{3} \sigma^{0} \delta_{r}}{2(4 \pi)^{3} k T_{0} F L R^{3} V \cos \psi}
$$

Where $P_{t}, G,(\lambda),(R), T_{0}, F, L, V$, and $(\psi)$ are the radar power transmit, gain of the antenna which is assumed to be identical between the transmitter and receiver, wavelenght, the distance between target and the observer, the total of noise temperature, the total noise figure of system, the total loss from the radar device, and velocity of the platform respectively. $(k)$ is Boltzmann constant which is $1.38 \times 10^{-23} \mathrm{JK}^{-1}$. This is ideally set to be .

\section{2..3 FMCW Signal Generation Using DDS}

Direct Digital Synthesizer (DDS) is a method to generate signal digitally. The DDS block system regularly consists of phase accumulator, phase to amplitude converter, digital to analog converter, and filter. The Fig . 4 shows the block system of a DDS [12].

The main part of a DDS system is on the phase accumulator. In this part, the bits that will be synthesized are sent into frequency register and phase register. The digital value that is coming out from the phase register will be delivered into the amplitude converter, that is ROM that contain sine look up table which is used to change the digital number from phase register output into sinusoidal signal in discrete form. The frequency of the DDS output can be determined using Eq.(11).

$$
f_{\text {out }}=\frac{M f_{c l k}}{2^{n}}
$$

Variable $M$ is the tuning word, $f_{c l k}$ is the reference clock, and $n$ is the bit of the phase accumulator. Based on Nyquist's Theorem, maximum frequency value that can be synthesized by DDS is half of the reference clock that is used by the DDS.

\section{FMCW-SAR Transmitter Design}

In this research, the part of FMCW-SAR that has been built is the FMCW-SAR transmitter. First, the radar performance that wants to be achieved will be explained then continue by explanation of the each component of the transmitter.

\section{3..1 Design of FMCW-SAR Performance}

The FMCW-SAR transmitter uses 2 DDS to produce complex signals. Both DDS is synchronized by using one reference clock. The complete block system of FMCW-SAR that has been researched is shown in Fig. 5. The complete transmitter specification is shown in Table 1.

\section{3..2 Design of FMCW-SAR Transmitter}

The designed FMCW-SAR transmitter is consist of chirp generator, up conversion block, and amplifier. The transmitter system is shown by Fig. 5.

The bill of material of this transmitter is listed in Table 2. In order to built a compact transmitter, the smaller components are intentionally picked up. The selected DDS module is the 
Edwar et al / Journal of Measurement, Electronics and Communication Systems

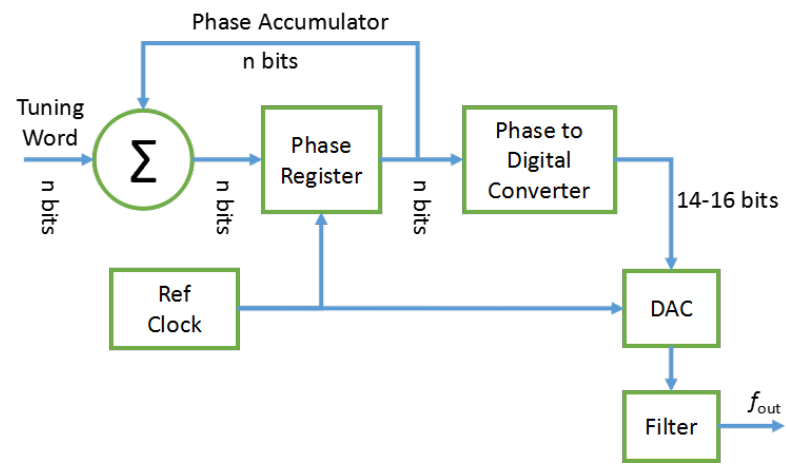

Fig. 4. Block System of DDS

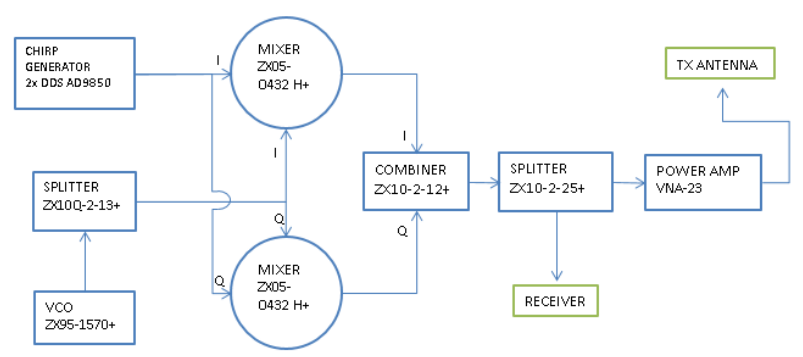

Fig. 5. Block System of SAR Transmitter

Table 1: FMCW-SAR Transmitter Specification

\begin{tabular}{|c||c|}
\hline Parameter & Value \\
\hline Frequency Carrier $\left(F_{0}\right)$ & $1.27 \mathrm{GHz}$ \\
\hline Start Frequency & $25 \mathrm{MHz}$ \\
\hline Stop Frequency & $35 \mathrm{MHz}$ \\
\hline Chirp Duration $(\tau)$ & $1 \mu s$ \\
\hline Bandwidth $(B)$ & $10 \mathrm{MHz}$ \\
\hline Frequency Deviation $(\Delta f)$ & $10 \mathrm{MHz}$ \\
\hline Resolution $(\Delta R)$ & $15 \mathrm{~m}$ \\
\hline
\end{tabular}

AD9850 and the microcontroller used is MBED LPC1768.

\section{3..3 The Simulation Result}

The FMCW chirp generation is the first important process in this system. To get the description of the FMCW chirp waveform, it needs to simulate the wanted system. The simulation to get the instantaneous phase of the FMCW signal is following both Eq.(12) and Eq.(13). The result of this simulation is the FMCW chirp at amplitude-time domain.

$$
f(t)=f_{0}+\mu t ; \quad-\frac{\tau_{0}}{2} \leq t \leq \frac{\tau_{0}}{2}
$$

Table 2: FMCW-SAR Components List

\begin{tabular}{|c||c|}
\hline \multicolumn{1}{|c||}{ Components } & Function \\
\hline AD9850 & DDS Module \\
\hline MBED LPC1768 & Microcontroller \\
\hline ZX-95-1570+ & VCO \\
\hline ZX10Q-2-13+ & 2-Way 90 Splitter \\
\hline ZX05-U432H+ & Mixer \\
\hline ZX10-2-25+ & Combiner \\
\hline VNA-23 & Power Amplifier \\
\hline
\end{tabular}

$$
f(t)=f_{0}-\mu t ; \quad-\frac{\tau_{0}}{2} \leq t \leq \frac{\tau_{0}}{2}
$$

The $f_{0}$ is the center frequency and $\mu$ is the LFM coefficient which is equal to $B / \mathrm{t}_{0}$ (Bandwidth divide by the chirp width). To investigate the triangle form of the FMCW chirp, the instantaneous frequency need to be simulated. The simulation to get the instantaneous frequency is done by following both Eq.(14) and Eq.(15). The result of this simulation is the signal FMCW in triangle form in frequency-time domain.

$$
\phi(t)=2 \pi\left(f_{0} t+\frac{\mu}{2} t^{2}\right) ; \quad-\frac{\tau_{0}}{2} \leq t \leq \frac{\tau_{0}}{2}
$$


Edwar et al / Journal of Measurement, Electronics and Communication Systems

$$
\phi(t)=2 \pi\left(f_{0} t-\frac{\mu}{2} t^{2}\right) ; \quad-\frac{\tau_{0}}{2} \leq t \leq \frac{\tau_{0}}{2}
$$

The result of all simulation is shown by Fig. 6 . The upper image is the FMCW chirp in amplitudetime domain, while the middle one is the triangle form of FMCW chirp in frequency-time domain, and the lower one is the up converted signal.
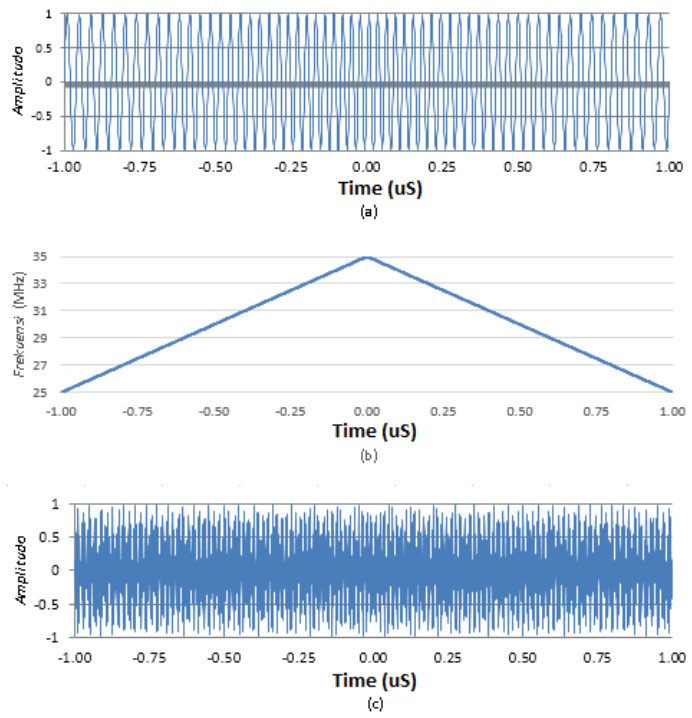

Fig. 6. The FMCW-SAR simulation result: (a) The Sinusoidal form of FMCW chirp; (b) Triangular FMCW; (c) The mixed signal

\section{Implementation And Analysis}

\section{4..1 Signal Generator Implementation}

As explained at the previous chapter, the transmitter uses two synchronized DDS to generate the FMCW chirp. Both module are connected to a microcontroller that constantly store the tuning word bit into both DDS. The communication used between microcontroller and the DDS is using SPI (Serial Peripheral Interface) bus. For the frequency update and reset triggers, two digital pins are utilized by sending a binary number that behave like a switch. The configuration is described by Fig. 7 and the FMCW generation result by using DDS is shown by Fig. 8 .

\section{4..2 Synchronization of Two DDS}

As explained before, to get inphase and quadrature in this FMCW-SAR system, the synchronization between two DDS has been done. The microcontroller is needed to store the tuning word to the both DDS with $90^{\circ}$ phase difference between them. The frequency updating process is intentionally delayed from their clock timing by using

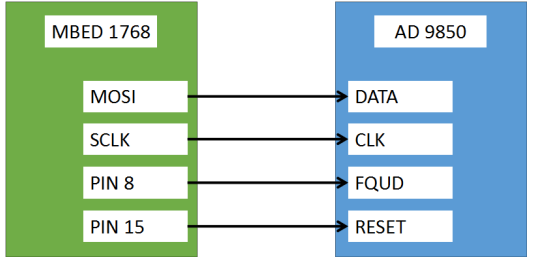

Fig. 7. DDS module test configuration

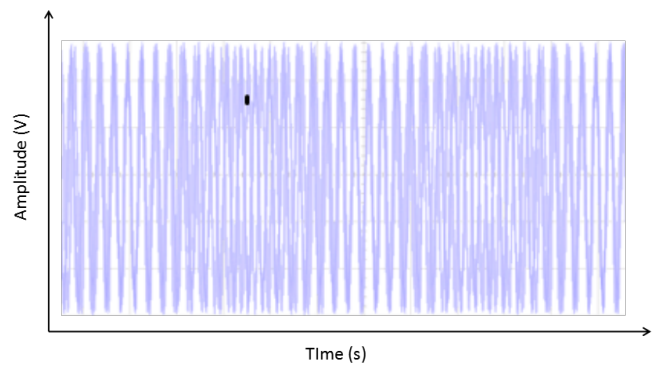

Fig. 8. DDS module testing result

D-Flip Flop. The purpose of delaying is to make the frequency updating valid by the DDS. To observe the synchronization result which produce the inphase and quarature signals, the output of each DDS is connected to oscilloscope. The result is shown by Fig. 9 .

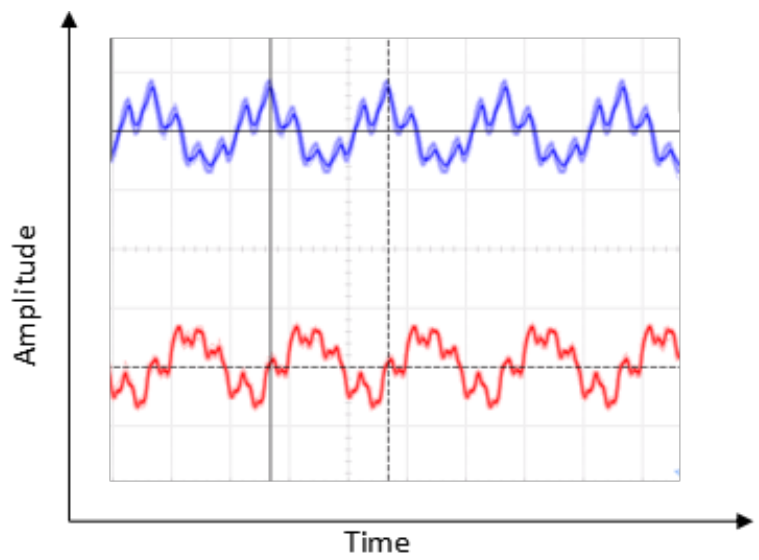

Fig. 9. The DDS synchronizaition result

The inphase and quadrature signal are succeed to be generated, but there was a mistake in the phase difference between both signals, where the wanted value was $90^{\circ}$ did not occurred. The actual phase value occurred is about $109.3^{\circ}$. This result has been analyzed and predicted to be caused by the asymmetric in the reference clock circuit path from the source, $125 \mathrm{MHz}$ crystal clock, to the both DDS and D-Flip Flop.

The DDS output also had been measured using the Spectrum Analyzer to observe how their spectrum are. The result is shown by Fig. 10 . 
Edwar et al / Journal of Measurement, Electronics and Communication Systems

The magnitude of both signal is slightly different on to each other, where the measured inphase signal magnitude was $-12.17 \mathrm{dBm}$, and quadrature signal was $-11.33 \mathrm{dBm}$.

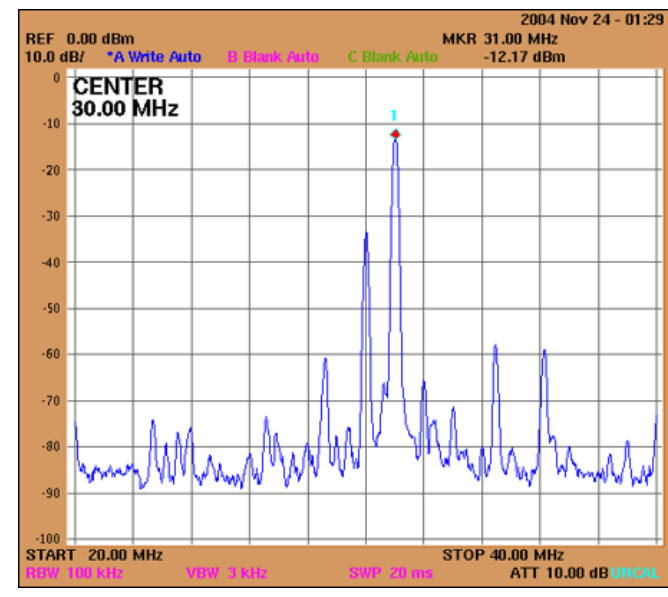

Fig. 10. Inphase spectrum at DDS output

\section{4..3 FMCW-SAR Payload Integration Result}

Integration has been done to produce the desired FMCW-SAR signal. This process has involved all the components in Table 2 . The construction of the FMCW-SAR payload follows the composition at Fig. 5. Furthermore, to regulate the carrier frequency on VCO, so that the module could generate the desired carrier frequency, LM2577 DC-DC Step Up module was used to produce right voltage to supply the $V_{\text {tune }}$ pin on VCO. The RF components integration results is shown in Fig. 11. These RF part will be equipped with the synchronized DDS module and power amplifier to build the complete FMCW-SAR transmitter. The Fig. 12 shows the result of the realization of the whole transmitter part. The measurement result is shown in Fig. 13 by using Spectrum Analyzer.

At the Fig. 14, the transmitted FMCW spectrum is successfully generated. However, the carrier signal also appears with the main spectrum which is indicated that this transmitter needs an additional RF filter to be implemented.

The magnitude of the FMCW signal is around $-18.37 \mathrm{dBm}$. This value include with the loss from the cable that used in measurement process about $0.7 \mathrm{dBm}$. So, the transmit power total, if it is subtracted by the cable loss, is $-17.67 \mathrm{dBm}$.

The performance of the FMCW-SAR transmitter has been investigated and concluded in the Tabel 3 with the assumption that this payload is installed on a Unmanned Aerial Vehicle (UAV) which fly on $5 \mathrm{~km}$ altitude with the velocity of 200 $\mathrm{km} / \mathrm{h}$. The result, by calculating all the variable

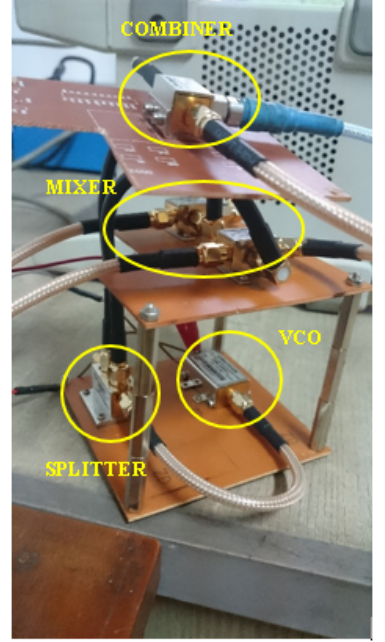

Fig. 11. Integration result for the RF part

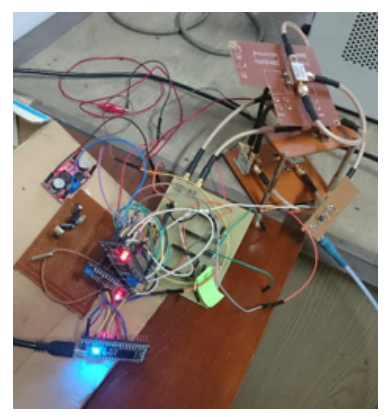

Fig. 12. FMCW-SAR tansmitter integration result

using Eq.(10), shows that the device will be expected to generate the $\mathrm{SNR}_{\text {out }}$ about $-42.63 \mathrm{~dB}$. Therefore, this transmitter output could be improved by using higher high power amplifier and antenna gain such as aperture antenna or array antenna.

\section{Conclusion}

We conclude that two DDS AD9850 has successfully produced inphase and quadrature signal. The measure of phase difference between those signal is $109.3^{\circ}$. For the future work, the length of circuit paths must be exactly the same to get the precision phase. The transmitter has been integrated and it has emitted the FMCW signal at 1.27 GHz with $10 \mathrm{MHz}$ bandwidth. FMCW-SAR transmit power that has been obtained in this research is $-17.67 \mathrm{dBm}$.

\section{Acknowledgment}

This work has been supported by National Graduate Program Scholarship for Prospective Lecturer from the Department of Education and 
Edwar et al / Journal of Measurement, Electronics and Communication Systems

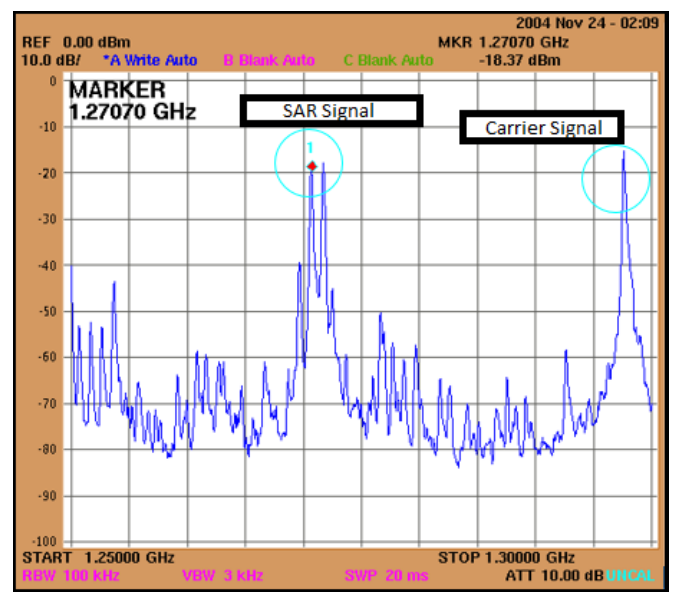

Fig. 13. FMCW-SAR tansmitter integration result

Technology, Indonesia.

\section{References}

[1] Edwar dan A. Munir, "Development of SAR Transmitter for Nanosatellite-based Remote Sensing Application", International Conference on Electrical Engineering and Informatics (ICEEI), 2015

[2] F. S. Marzano, S. Marchiotto, C. Textor, and D. J. Schneider, "Model-based weather radar remote sensing of explosive volcanic ash eruption", IEEE Transactions On Geoscience and Remote Sensing, Vol. 48, No. 10, October 2010, pp. 3591-3607

[3] F. Tessitore, "Aerial surveillance and airborne remote sensing techniques in Guardia di Finanza: an operational experience", International Carnahan Conference on Security Technology (ICCST), 2014, pp. 1-6

[4] Y. Zhou, S. Wang, W. Zhou, P. Zhang, "Applications of CBERS-2 image data in flood disaster remote sensing monitoring", International Geoscience and Remote Sensing Symposium (IGARSS '04), Vol. 7, 2004, pp. 4696-4699

[5] J. T. S. Sumantyo, "Development of circularly polarized synthetic aperture radar onboard microsatellite for earth diagnosis", International Geoscience and Remote Sensing Symposium (IGARSS), 2011, pp. 929-932

[6] A. Moreira, P.P. Siraola, M. Younis, G. Krieger, I. Hajnsek, and K.P. Papathanassiou, "A tutorial on synthethic aperture radar", IEEE Geoscience and Remote Sensing Magazine, Vol. 1, March 2013, pp. 6-43
Table 3: FMCW-SAR transmitter integration result

\begin{tabular}{|c||c|}
\hline Parameter & Value \\
\hline Power transmit $\left(P_{t}\right)$ & $-17.67 \mathrm{dBm}$ \\
\hline Antenna gain $(G)$ & $8 \mathrm{~dB}$ \\
\hline Lamdha $(\lambda)$ & $-6.27 \mathrm{~dB}$ \\
\hline RCS per unit $\left(\sigma^{0}\right)$ & $-16.63 \mathrm{~dB}$ \\
\hline Boltzmann constant $(k)$ & $-228.601 \mathrm{~dB}$ \\
\hline Temperature noise $\left(T_{e}\right)$ & $24.62 \mathrm{~dB}$ \\
\hline Loss $(L)$ & $2 \mathrm{~dB}$ \\
\hline Noise figure $(F)$ & $4.7 \mathrm{~dB}$ \\
\hline Pixel size $\left(\delta_{r}\right)$ & $17.32 \mathrm{~m}$ \\
\hline Platform velocity $(V)$ & $200 \mathrm{~km} / \mathrm{h}$ \\
\hline Grazzling angle $(\psi)$ & $30^{\circ}$ \\
\hline Maximum range $(R)$ & $10000 \mathrm{~m}$ \\
\hline Altitude $(H)$ & $5000 \mathrm{~m}$ \\
\hline SNR & $\mathbf{- 4 2 . 6 3 d B}$ \\
\hline
\end{tabular}

[7] https://histru.bournemouth.ac.uk/OralHistory/Talking-About-Technology/radarresearch/chain-home-operational.html, diakses pada 12 Desember 2015

[8] M. Mahrooghy, J. Aanstoos, K. Hasan, S. Prasad, N. H. Younan., "Effect of vegetation height and volume scattering on soil moisture classification using synthetic aperture radar (SAR) images", Applied Imagery Pattern Recognition Workshop (AIPR), 2011, pp. $1-5$

[9] W. Wayne, Introduction to Radar Remote Sensing for Vegetation Mapping and Monitoring

[10] B. R. Mahafza, Radar Systems Analysis and Design Using MATLAB, $3^{\text {rd }}$ Edition, CRC Press, 2012

[11] M. Skolnik, Radar Handbook, $3^{\text {rd }}$ Ed, Mc Graw Hill, 2008

[12] E. Murphy, C. Slattery, All About Direct Digital Synthesis, Analog Dialogue 38-08, 2008 
Edwar et al / Journal of Measurement, Electronics and Communication Systems

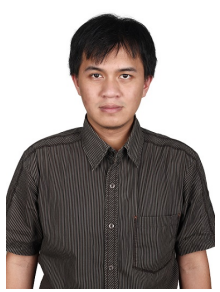

Edwar, S.T, M.T. He finished his bachelor degree in Telkom University, then continued the master degree in Bandung Institute of Technology. Currently he is a lecturer in the School of Electrical Engineering Telkom University. He is also the Head of Electronic of Telecommunication Laboratory and also the member of Nanosatellite Laboratory in Telkom University. He has interest on Nanosatellite subsystem and payload research, RF device and its application especially in remote sensing area and has been published some papers and journals in those fields on international proceeding and national journal.

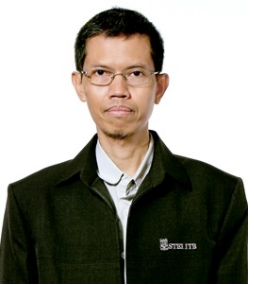

Dr.Eng. Achmad Munir, S.T., M.Eng. He received the D.E. degree from Yamaguchi University, Japan, in 2005. From 2005 to 2007, he was a Research Fellow under JSPS fellowship program with department of Electrical and Electronics Engineering, Faculty of Engineering, Yamaguchi University, Japan. From 2007 to 2009, he was a Research Fellow with the Institute of Electronics, Communications, and Information Technology, Queens University Belfast, Northern Ireland, United Kingdom. Currently he is an associate professor at the School of Electrical Engineering and Informatics, Institut Technology of Bandung, Indonesia. He has interest on RF devices including metamaterial substrate characteristic. He has contributed in so many research within those fields and produced so many papers and journals in many prestigious international conferences and journals and granted as the most productive indonesian researcher at 2017. 